\title{
Inertial hybrid S-iteration algorithm for fixed point of asymptotically nonexpansive mappings and equilibrium problems in a real Hilbert space
}

\author{
M. H. Harbau ${ }^{\mathrm{a}, *}$, Abdulwahab Ahmad ${ }^{\mathrm{b}}$ \\ ${ }^{a}$ Department of Science and Technology Education, Bayero University, Kano, Nigeria. \\ ${ }^{b}$ Department of Mathematics, Federal College of Education, Katsina, Nigeria.
}

\begin{abstract}
In this paper, we introduce an inertial hybrid S-iteration algorithm for two asymptotically nonexpansive mappings and equilibrium problems in a real Hilbert space. Strong convergence of the iterative scheme is established. Our results improve and extend many recent results in the literature.
\end{abstract}

Keywords: Asymptotically nonexpansive, inertial S-iteration method, equilibrium problems, fixed point.

2020 MSC: 47H09, 47J25.

(C)2022 All rights reserved.

\section{Introduction}

Let $\mathrm{C}$ be a nonempty closed and convex subset of a real Hilbert space $\mathrm{H}$, with an inner product $\langle.,$. and induced norm $\|$.$\| on \mathrm{H}$. The equilibrium problem (shortly written as EP), see [4, 25], is a problem of finding $x^{*} \in \mathrm{C}$, such that

$$
f\left(x^{*}, y\right) \geqslant 0, \quad \forall y \in C,
$$

where $f$ is a bifuntion on $C \times C$. We denote by $\operatorname{EP}(f, C)$ the set of solutions of equilibrium problem (1.1), i.e., $\operatorname{EP}(f, C)=\left\{x^{*} \in C: f\left(x^{*}, y\right) \geqslant 0, \forall y \in C\right\}$. Let $B: C \rightarrow H$ be a map. The variational inequality problem with respect to $B$, defined on $C$ is to find a point $z \in C$, such that

$$
\langle\mathrm{B} z, \mathrm{y}-z\rangle \geqslant 0, \quad \forall \mathrm{y} \in \mathrm{C} .
$$

The set of solutions of problem (1.2) is denoted by $\operatorname{VI}(C, B)$, i.e., $\operatorname{VI}(C, B)=\{z \in C:\langle B z, y-z\rangle \geqslant 0, \forall y \in$ $C\}$. Setting $f(z, y)=\langle B z, y-z\rangle, \forall y \in C$, then, $z \in E P(f, C)$ if and only if $\langle B z, y-z\rangle \geqslant 0, \forall y \in C$. That is $z$ is a solution of problem (1.2).

Let $S: C \rightarrow C$ be a nonlinear map. The fixed point problem with respect to $S$ is to find a point $x \in C$ such that $S x=x$. We denote by $\operatorname{Fix}(S)$ the set of all fixed points of $S$, i.e., $\operatorname{Fix}(S)=\{x \in C: S x=x\}$. If the

\footnotetext{
*Corresponding author

Email addresses: murtalaharbau@yahoo.com (M. H. Harbau), abumuhammaddm@gmail.com (Abdulwahab Ahmad)

doi: $10.22436 /$ jnsa.015.02.04
}

Received: 2021-07-21 Revised: 2021-08-11 Accepted: 2021-10-07 
map S satisfies the following condition,

$$
\|S x-S y\| \leqslant k\|x-y\|, \quad \forall x, y \in C,
$$

where $k$ is some constant in $[0,1)$, then it is called a contraction. However, if $k=1$ in (1.3), then $S$ is nonexpansive. $S$ is asymptotically nonexpansive (see [10]) if there exists a sequence $\left\{k_{n}\right\} \subset[0, \infty)$, with $\lim _{n \rightarrow \infty} k_{n}=0$ such that

$$
\left\|S^{n} x-S^{n} y\right\| \leqslant\left(1+k_{n}\right)\|x-y\|, \quad \forall x, y \in C n \geqslant 1 .
$$

$S$ is uniformly L-Lipschitzian if there exists a constant $L>0$ such that, for all $x, y \in C$,

$$
\left\|S^{n} x-S^{n} y\right\| \leqslant L\|x-y\|, \quad \forall n \geqslant 1 .
$$

Remark 1.1. It can easily be seen from the above that the class of nonexpansive mappings is properly contained in the class of asymptotically nonexpansive mappings and every asymptotically nonexpansive mapping is uniformly L-Lipschitzian, for more details see Goebel and Kirk [10].

The equilibrium problems and fixed point problems, which are closely related are problems that arise in various applications, such as in optimization, physics, economics, engineering, theory of differential equations, game theory, image recovery, signal processing, and other important areas in mathematical sciences, (see, for examples, [4, 16, 17, 19, 26]). Various methods have been proposed for approximating solutions of fixed point problems and equilibrium problems in various spaces (see for examples [2$4,14,15,18,19,24,26,31,33-35]$ and the references therein).

Takahashi et al. [36] introduce a hybrid method for nonexpansive mapping called shrinking projection method in Hilbert space as follows;

$$
\left\{\begin{array}{l}
x_{0} \in H, \\
C_{1}=C, x_{1}=P_{C_{1}} x_{0}, \\
y_{n}=\alpha_{n} x_{n}+\left(1-\alpha_{n}\right) T x_{n}, \\
C_{n+1}=\left\{z \in C_{n}:\left\|y_{n}-z\right\| \leqslant\left\|x_{n}-z\right\|\right\}, \\
x_{n+1}=P_{C_{n+1}} x_{0}, \quad n \geqslant 1,
\end{array}\right.
$$

where $0 \leqslant \alpha_{n} \leqslant a<1, \forall n \geqslant 1$. They proved strong convergence of (1.4) to fixed point of $T$.

Agarwal et al. [1] introduced an iterative method of the same convergence rate with Picard algorithm in [28], called S-iteration method as follows;

$$
\left\{\begin{array}{l}
x_{0} \in C \\
y_{n}=\left(1-\beta_{n}^{1}\right) x_{n}+\beta_{n}^{1} S x_{n}, \\
x_{n+1}=\left(1-\beta_{n}^{0}\right) S x_{n}+\beta_{n}^{0} S y_{n}, \quad n \geqslant 1
\end{array}\right.
$$

they proved that it converges faster than the Mann algorithm in [22], for the class of maps satisfying (1.3).

Sahu [30] proved both theoretically and numerically that S-iteration method (1.5) converges faster than both Picard algorithm in [28] and Mann algorithm in [22] for contraction mappings.

Recently, Suparatulatorn et al. [32] proved weak and strong convergence S-iteration process for two G-nonexpansive mappings $S_{1}$ and $S_{2}$ in Hilbert space as follows:

$$
\left\{\begin{array}{l}
x_{0} \in C \\
y_{n}=\left(1-\beta_{n}\right) x_{n}+\beta_{n} S_{1} x_{n}, \\
x_{n+1}=\left(1-\alpha_{n}\right) S_{1} x_{n}+\alpha_{n} S_{2} y_{n}, \quad n \geqslant 0
\end{array}\right.
$$

However, there have been tremendous interests in developing fast convergence algorithms, especially for the inertial type algorithms, which was first proposed by Polyak in [29] as an acceleration process in solving a smooth convex minimization problem. Recently, some researchers have constructed various fast 
iterative algorithms by inertial extrapolation techniques (see $[5-7,9,12,13,16,17,20]$ and the references contained therein).

Mainge [21] proposed the following inertial Mann algorithm by combining the Mann algorithm in [22] and inertial extrapolation.

$$
\left\{\begin{array}{l}
w_{n}=x_{n}+\gamma_{n}\left(x_{n}-x_{n-1}\right) \\
x_{n+1}=\left(1-\alpha_{n}\right) w_{n}+\alpha_{n} T w_{n}, \quad n \geqslant 1
\end{array}\right.
$$

He proved a weak convergence theorem for the scheme (1.7) under some conditions on the sequences of parameters $\left\{\gamma_{n}\right\},\left\{\alpha_{n}\right\}$.

Very recently, Phon-on et al. [27] proposed the following modified inertial S-iteration process by combining (1.6) and the inertial extrapolation, and consequently obtain the following accelerated approximation method for two nonexpansive mappings $S_{1}$ and $S_{2}$ in Hilbert space.

$$
\left\{\begin{array}{l}
x_{0}, x_{1} \in H, \\
w_{n}=x_{n}+\gamma_{n}\left(x_{n}-x_{n-1}\right), \\
y_{n}=\left(1-\beta_{n}\right) w_{n}+\beta_{n} S_{1} w_{n}, \\
x_{n+1}=\left(1-\alpha_{n}\right) S_{1} w_{n}+\alpha_{n} S_{2} y_{n}, \quad n \geqslant 1,
\end{array}\right.
$$

where $\left\{\gamma_{n}\right\},\left\{\alpha_{n}\right\}$, and $\left\{\beta_{n}\right\}$ satisfy some conditions.

Inspired and motivated by the results of $[27,32,36]$, our purpose in this paper is to introduce an inertial hybrid S-iteration algorithm for solving monotone equilibrium problem and fixed point problem of two asymptotically nonexpansive mappings in a real Hilbert space. We also give numerical examples to justify that our scheme is more effective and implementable. Our results generalize and improve recent results in the literature.

\section{Preliminaries}

Let $\mathrm{H}$ be a real Hilbert space. The following identity is well known

$$
\|\lambda x+(1-\lambda) y\|^{2}=\lambda\|x\|^{2}+(1-\lambda)\|y\|^{2}-\lambda(1-\lambda)\|x-y\|^{2}, \quad \forall x, y \in H, \lambda \in \mathbb{R} .
$$

It is also known that for any $x \in H$, there exists a unique element denoted by $P_{C} x$ in $C$, such that

$$
\left\|x-P_{C} x\right\| \leqslant\|x-y\|, \quad \forall y \in C .
$$

The mapping $\mathrm{P}_{\mathrm{C}}$ is called the metric projection from $\mathrm{H}$ onto $\mathrm{C}$. In addition, $\mathrm{P}_{\mathrm{C}}$ has the following characteristics, (see, for example Goebel and Reich [11]):

(i) $\left\langle x-y, P_{C} x-P_{C} y\right\rangle \geqslant\left\|P_{C} x-P_{C} y\right\|^{2}, \forall x, y \in H$;

(ii) for $x \in H$, and $x^{*} \in C$,

$$
x^{*}=P_{C} x, \Leftrightarrow\left\langle x-x^{*}, x^{*}-y\right\rangle \geqslant 0, \forall y \in C ;
$$

(iii) for $x \in H$ and $y \in C$,

$$
\left\|x-P_{C} x\right\|^{2}+\left\|y-P_{C} x\right\|^{2} \leqslant\|x-y\|^{2} .
$$

Definition 2.1. A nonlinear map $S$ is said to be demiclosed at $y_{0} \in X$, if for any sequence $\left\{x_{n}\right\}$ in $X$ which converges weakly to $x_{0} \in X$ and $S x_{n} \rightarrow y_{0}$, it holds that $S x_{0}=y_{0}$.

To solve the equilibrium problem (1.1), we assume the bifunction $f$ to satisfies the following conditions:

$\left(\mathrm{G}_{1}\right) \mathrm{f}(\mathrm{x}, \mathrm{x})=0, \quad \forall x \in \mathrm{C}$;

$\left(G_{2}\right) f$ is monotone, i.e., $f(x, y)+f(y, x) \leqslant 0, \quad \forall x, y \in C$;

(G) for each $x, y, z \in C$;

$$
\lim _{s \rightarrow 0} f(s z+(1-s) x, y) \leqslant f(x, y)
$$


$\left(G_{4}\right)$ for each $x \in C, y \longmapsto f(x, y)$ is convex and lower semi continuous.

The following Lemmas will be needed in the proof of the main results.

Lemma 2.2 ([38]). Let $\mathrm{X}$ be a real uniformly convex Banach space and $\mathrm{C}$ be a nonempty closed and convex subset of $\mathrm{X}$. Let $\mathrm{T}: \mathrm{C} \rightarrow \mathrm{C}$ be asymptotically nonexpansive mapping with sequence $\left\{\mathrm{k}_{\mathrm{n}}\right\} \subset[0, \infty)$ such that $\lim _{\mathrm{n} \rightarrow \infty} \mathrm{k}_{\mathrm{n}}=0$. Then, the mapping $(\mathrm{I}-\mathrm{T})$ is demiclosed at zero.

Lemma 2.3 ([10]). Let $\mathrm{C}$ be a nonempty closed and convex subset of a uniformly convex Banach space $\mathrm{X}$, and let $\mathrm{T}: \mathrm{C} \rightarrow \mathrm{C}$ be asymptotically nonexpansive map. Then, $\mathrm{F}(\mathrm{T})$ is closed and convex.

Lemma 2.4 ([23]). Let $\mathrm{C}$ be a closed convex subset of $\mathrm{H}$. Let $\left\{\mathrm{x}_{\mathrm{n}}\right\}$ be a sequence in $\mathrm{H}$ and $\mathrm{u} \in \mathrm{H}$. Let $\mathrm{x}^{*}=\mathrm{P}_{\mathrm{C}} \mathrm{u}$. If $\left\{x_{n}\right\}$ is such that $\omega_{w}\left(x_{n}\right) \subset C$ and satisfies the condition

$$
\left\|x_{n}-u\right\| \leqslant\left\|u-x^{*}\right\|, \quad \forall n \geqslant 1,
$$

then $x_{n} \rightarrow x^{*}$, where $\omega_{w}\left(x_{n}\right)=\left\{y \in C: \exists\left\{x_{n_{j}}\right\} \subset\left\{x_{n}\right\}\right.$ and $\left.x_{n_{j}} \rightarrow y\right\}$.

Lemma 2.5 ([4]). Let $\mathrm{C}$ be a nonempty closed and convex subset of $\mathrm{H}$ and $\mathrm{f}$ be a bifunction of $\mathrm{C} \times \mathrm{C}$ into $\mathbb{R}$ satisfying $\left(\mathrm{G}_{1}\right)-\left(\mathrm{G}_{4}\right)$. Let $\mathrm{r}>\mathrm{o}$ and $\mathrm{x} \in \mathrm{H}$. Then, there exists $z \in \mathrm{C}$, such that

$$
f(z, y)+\frac{1}{r}\langle y-z, z-x\rangle \geqslant 0, \forall y \in C .
$$

Lemma 2.6 ([8, 37]). Assume that $\mathrm{f}: \mathrm{C} \times \mathrm{C} \rightarrow \mathbb{R}$ satisfies $\left(\mathrm{G}_{1}\right)-\left(\mathrm{G}_{4}\right)$. For $\mathrm{r}>0$ and $\mathrm{x} \in \mathrm{H}$, define a mapping $\mathrm{T}_{\mathrm{r}}: \mathrm{H} \rightarrow \mathrm{C}$ as follows:

$$
\mathrm{T}_{\mathrm{r}}(x)=\left\{z \in \mathrm{C}: \mathrm{f}(z, \mathrm{y})+\frac{1}{\mathrm{r}}\langle\mathrm{y}-z, z-x\rangle \geqslant 0, \forall \mathrm{y} \in \mathrm{C}\right\}
$$

for all $\mathrm{x} \in \mathrm{H}$. Then, the following hold:

(i) $\mathrm{T}_{\mathrm{r}}$ is single-valued;

(ii) $\mathrm{T}_{\mathrm{r}}$ is firmly nonexpansive, i.e., for $\mathrm{x}, \mathrm{y} \in \mathrm{H},\left\|\mathrm{T}_{\mathrm{r}} \mathrm{x}-\mathrm{T}_{\mathrm{r}} \mathrm{y}\right\|^{2} \leqslant\left\langle\mathrm{~T}_{\mathrm{r}} x-\mathrm{T}_{\mathrm{r}} \mathrm{y}, \mathrm{x}-\mathrm{y}\right\rangle$;

(iii) $\mathrm{F}\left(\mathrm{T}_{\mathrm{r}}\right)=\mathrm{EP}(\mathrm{f})$;

(iv) $\mathrm{EP}(\mathrm{f})$ is closed and convex;

(v) $\left\|q-T_{r} x\right\|^{2}+\left\|T_{r} x-x\right\|^{2} \leqslant\|q-x\|^{2}, \quad \forall q \in F\left(T_{r}\right), x \in H$.

\section{Main result}

Theorem 3.1. Let $\mathrm{H}$ be a real Hilbert space and $\mathrm{C}$ be a nonempty closed, bounded and convex subset of $\mathrm{H}$. Let $f: C \times C \rightarrow \mathbb{R}$ be a bifunction satisfying conditions $\left(\mathrm{G}_{1}\right)-\left(\mathrm{G}_{4}\right)$. Let $\mathrm{T}_{i}: \mathrm{C} \rightarrow \mathrm{C}, \mathrm{i}=1,2$, be asymptotically nonexpansive mapping with sequence $\left\{k_{n, i}\right\} \subset[0, \infty)$ such that $\lim _{n \rightarrow \infty} k_{n, i}=0$ and $\bigcap_{i=1}^{2} F\left(T_{i}\right) \cap E P(f, C) \neq \emptyset$. For $\mathrm{x}_{0}, \mathrm{x}_{1} \in \mathrm{C}$ and $\mathrm{C}_{0}=\mathrm{C}$, let $\left\{\mathrm{x}_{\mathrm{n}}\right\}$ be a sequence generated by;

$$
\left\{\begin{array}{l}
w_{n}=x_{n}+\alpha_{n}\left(x_{n}-x_{n-1}\right) \\
y_{n}=\left(1-\beta_{n}\right) w_{n}+\beta_{n} T_{1}^{n} w_{n}, \\
z_{n}=\left(1-\gamma_{n}\right) T_{1}^{n} w_{n}+\gamma_{n} T_{2}^{n} y_{n}, \\
u_{n}=T_{r_{n}} z_{n}, \\
C_{n+1}=\left\{z \in C_{n}:\left\|u_{n}-z\right\|^{2} \leqslant\left\|w_{n}-z\right\|^{2}+\theta_{n}\right\} \\
x_{n+1}=P_{C_{n+1}} x_{0}, \quad \forall n \geqslant 0,
\end{array}\right.
$$

where $\theta_{n}=\left(\left(2 k_{n, 1}+k_{n, 1}^{2}\right)\left(1+\gamma_{n} \beta_{n}\right)+\gamma_{n}\left(2 k_{n, 2}+k_{n, 2}^{2}\right)\left(1+\beta_{n}\left(2 k_{n, 1}+k_{n, 1}^{2}\right)\right)\right) M^{2}, M=\operatorname{diamC}=$ sup $\|x-y\|$ and $\left\{r_{n}\right\} \subset[a, \infty)$ for some $a>0$. Then, the sequence $\left\{x_{n}\right\}$ converges strongly to a point $x, y \in C$

$x \in \bigcap_{i=1}^{2} F\left(T_{i}\right) \cap \operatorname{EP}(f, C)$, provided that the sequences of real numbers $\left\{\alpha_{n}\right\} \subset(0,1),\left\{\beta_{n}\right\}$ and $\left\{\gamma_{n}\right\}$ satisfy the following condition: 
(C1) $\beta_{n}, \gamma_{n} \in[\zeta, 1-\zeta]$, for some $\zeta \in(0,1)$.

Proof. We divide the proof into the following steps.

Step (i): We show that $\left\{x_{n}\right\}$ is well defined and $\bigcap_{i=1}^{2} F\left(T_{i}\right) \cap \operatorname{EP}(f, C) \subset C_{n}, \forall n \geqslant 0$.

From the scheme, $\mathrm{C}_{0}=\mathrm{C}$ is closed and convex. We also observe that

$$
\left\|u_{n}-z\right\|^{2} \leqslant\left\|w_{n}-z\right\|^{2}+\theta_{n} \quad \Leftrightarrow \quad 2\left\langle z, w-u_{n}\right\rangle \leqslant\left\|w_{n}\right\|^{2}-\left\|u_{n}\right\|^{2}+\theta_{n} .
$$

Therefore, from (3.2), we have that $C_{n}$ is closed and convex, $\forall n \geqslant 0$, and consequently $\left\{x_{n}\right\}$ is well defined. It follows from Lemma 2.3 that for each $i=1,2, F\left(T_{i}\right)$ is closed and convex, which implies that $\bigcap_{i=1}^{2} F\left(T_{i}\right)$ is closed and convex. It also follows from Lemma 2.6 (iv) that $\operatorname{EP}(f, C)$ is closed and convex. Consequently, $\bigcap_{i=1}^{2} F\left(T_{i}\right) \cap E P(f, C)$ is closed and convex. Thus, $P_{\bigcap_{i=1}^{2} F\left(T_{i}\right) \cap E P(f, C)} x_{0}$ is well defined.

Observe that $\bigcap_{i=1}^{2} F\left(T_{i}\right) \cap \operatorname{EP}(f, C) \subset C=C_{0}$. Assume $\bigcap_{i=1}^{2} F\left(T_{i}\right) \cap \operatorname{EP}(f, C) \subset C_{n}$ and let $p \in$ $\bigcap_{i=1}^{2} F\left(T_{i}\right) \cap E P(f, C)$, then by convexity of $\|.\|^{2}$, we have

$$
\begin{aligned}
\left\|y_{n}-p\right\|^{2} & =\left\|\left(1-\beta_{n}\right) w_{n}+\beta_{n} T_{1}^{n} w_{n}-p\right\|^{2} \\
& =\left\|\left(1-\beta_{n}\right)\left(w_{n}-p\right)+\beta_{n}\left(T_{1}^{n} w_{n}-p\right)\right\|^{2} \\
& \leqslant\left(1-\beta_{n}\right)\left\|w_{n}-p\right\|^{2}+\beta_{n}\left\|T_{1}^{n} w_{n}-p\right\|^{2} \\
& \leqslant\left(1-\beta_{n}\right)\left\|w_{n}-p\right\|^{2}+\beta_{n}\left(1+k_{n, 1}\right)^{2}\left\|w_{n}-p\right\|^{2} \\
& =\left\|w_{n}-p\right\|^{2}+\beta_{n}\left(2 k_{n, 1}+k_{n, 1}^{2}\right)\left\|w_{n}-p\right\|^{2} .
\end{aligned}
$$

Putting $u_{n}=T_{r_{n}} z_{n}$, using (3.3) and Lemma 2.6 (ii), we have

$$
\begin{aligned}
\left\|u_{n}-p\right\|^{2}= & \left\|T_{r_{n}} z_{n}-p\right\|^{2} \\
\leqslant & \left\|z_{n}-p\right\|^{2} \\
= & \left\|\left(1-\gamma_{n}\right) T_{1}^{n} w_{n}+\gamma_{n} T_{2}^{n} y_{n}-p\right\|^{2} \\
= & \left\|\left(1-\gamma_{n}\right)\left(T_{1}^{n} w_{n}-p\right)+\gamma_{n}\left(T_{2}^{n} y_{n}-p\right)\right\|^{2} \\
\leqslant & \left(1-\gamma_{n}\right)\left\|T_{1}^{n} w_{n}-p\right\|^{2}+\gamma_{n}\left\|T_{2}^{n} y_{n}-p\right\|^{2} \\
\leqslant & \left(1-\gamma_{n}\right)\left\|w_{n}-p\right\|^{2}+\left(2 k_{n, 1}+k_{n, 1}^{2}\right)\left\|w_{n}-p\right\|^{2}+\gamma_{n}\left(1+k_{n, 2}\right)^{2}\left\|y_{n}-p\right\|^{2} \\
\leqslant & \left(1-\gamma_{n}\right)\left\|w_{n}-p\right\|^{2}+\left(2 k_{n, 1}+k_{n, 1}^{2}\right)\left\|w_{n}-p\right\|^{2} \\
& +\gamma_{n}\left(1+k_{n, 2}\right)^{2}\left(\left\|w_{n}-p\right\|^{2}+\beta_{n}\left(2 k_{n, 1}+k_{n, 1}^{2}\right)\left\|w_{n}-p\right\|^{2}\right) \\
= & \left(1-\gamma_{n}\right)\left\|w_{n}-p\right\|^{2}+\left(2 k_{n, 1}+k_{n, 1}^{2}\right)\left\|w_{n}-p\right\|^{2} \\
& +\gamma_{n}\left(\left\|w_{n}-p\right\|^{2}+\beta_{n}\left(2 k_{n, 1}+k_{n, 1}^{2}\right)\left\|w_{n}-p\right\|^{2}\right) \\
& +\gamma_{n}\left(2 k_{n, 2}+k_{n, 2}^{2}\right)\left(\left\|w_{n}-p\right\|^{2}+\beta_{n}\left(2 k_{n, 1}+k_{n, 1}^{2}\right)\left\|w_{n}-p\right\|^{2}\right) \\
= & \left(1-\gamma_{n}\right)\left\|w_{n}-p\right\|^{2}+\left(2 k_{n, 1}+k_{n, 1}^{2}\right)\left\|w_{n}-p\right\|^{2}+\gamma_{n}\left\|w_{n}-p\right\|^{2} \\
& +\gamma_{n} \beta_{n}\left(2 k_{n, 1}+k_{n, 1}^{2}\right)\left\|w_{n}-p\right\|^{2}+\gamma_{n}\left(2 k_{n, 2}+k_{n, 2}^{2}\right)\left(1+\beta_{n}\left(2 k_{n, 1}+k_{n, 1}^{2}\right)\right)\left\|w_{n}-p\right\|^{2} \\
= & \left\|w_{n}-p\right\|^{2}+\left(2 k_{n, 1}+k_{n, 1}^{2}\right)\left(1+\gamma_{n} \beta_{n}\right)\left\|w_{n}-p\right\|^{2} \\
& +\gamma_{n}\left(2 k_{n, 2}+k_{n, 2}^{2}\right)\left(1+\beta_{n}\left(2 k_{n, 1}+k_{n, 1}^{2}\right)\right)\left\|w_{n}-p\right\|^{2} \\
\leqslant & \left\|w_{n}-p\right\|^{2}+\left(2 k_{n, 1}+k_{n, 1}^{2}\right)\left(1+\gamma_{n} \beta_{n}\right) M^{2}+\gamma_{n}\left(2 k_{n, 2}+k_{n, 2}^{2}\right)\left(1+\beta_{n}\left(2 k_{n, 1}+k_{n, 1}^{2}\right)\right) M^{2} \\
= & \left\|w_{n}-p\right\|^{2}+\theta_{n} .
\end{aligned}
$$

Hence, we have that $p \in C_{n+1}$. This implies that $\bigcap_{i=1}^{2} F\left(T_{i}\right) \cap E P(f, C) \subset C_{n+1}$. Thus, $\bigcap_{i=1}^{2} F\left(T_{i}\right) \cap$ $\mathrm{EP}(\mathrm{f}, \mathrm{C}) \subset \mathrm{C}_{\mathrm{n}}, \forall \mathrm{n} \geqslant 0$. 
Step (ii): We show that $\lim _{n \rightarrow \infty}\left\|x_{n}-x_{0}\right\|$ exists.

Since $x_{n}=P_{C_{n}} x_{0}$, and $x_{n+1} \in C_{n+1} \in C_{n}, \forall n \geqslant 0$, we have

$$
\left\|x_{n}-x_{0}\right\|=\left\|P_{C_{n}} x_{0}-x_{0}\right\| \leqslant\left\|x_{n+1}-x_{0}\right\|, \quad \forall n \geqslant 0,
$$

showing that $\left\{\left\|x_{n}-x_{0}\right\|\right\}$ is nondecreasing sequence. We also obtain from the definition of $x_{n}$ and (2.2) that

$$
\begin{aligned}
\left\|x_{n}-x_{0}\right\|^{2} & =\left\|P_{C_{n}} x_{0}-x_{0}\right\|^{2} \\
& \leqslant\left\|p-x_{0}\right\|^{2}-\left\|p-P_{C_{n}} x_{0}\right\|^{2} \\
& =\left\|p-x_{0}\right\|^{2}-\left\|p-x_{n}\right\|^{2} \\
& \leqslant\left\|p-x_{0}\right\|^{2}, \quad \forall p \in \bigcap_{i=1}^{2} F\left(T_{i}\right) \cap E P(f, C), p=P_{\bigcap_{i=1}^{2} F\left(T_{i}\right) \cap E P(f, C)} x_{0} .
\end{aligned}
$$

Therefore, $\left\{\left\|x_{n}-x_{0}\right\|\right\}$ is bounded. Consequently, $\left\{x_{n}\right\}$ is bounded. From (3.4) and (3.5), we get that $\lim _{n \rightarrow \infty}\left\|x_{n}-x_{0}\right\|$ exists.

Step (iii): We show that $\lim _{n \rightarrow \infty}\left\|x_{n}-T_{i} x_{n}\right\|=0$, for $i=1,2$.

From $x_{m}=P_{C_{m}} x_{0} \in \stackrel{n \rightarrow \infty}{C_{m}} \in C_{n}$, for $m>n$, then by (2.2), we get that

$$
\left\|x_{m}-x_{n}\right\|^{2} \leqslant\left\|x_{n}-x_{0}\right\|^{2}-\left\|x_{m}-x_{0}\right\|^{2} \rightarrow 0 \text { as } n \rightarrow \infty .
$$

Hence, $\left\{x_{n}\right\}$ is Cauchy in $H$.

Similarly, since $x_{n+1} \in P_{C_{n+1}} x_{0} \in C_{n+1} \subset C_{n}$, then

$$
\left\|x_{n+1}-\left.x_{n}\right|^{2} \leqslant\right\| x_{n}-x_{0}\left\|^{2}-\right\| x_{n+1}-x_{0} \|^{2} .
$$

Since $\lim _{n \rightarrow \infty}\left\|x_{n}-x_{0}\right\|$ exists, we obtain

$$
\lim _{n \rightarrow \infty}\left\|x_{n+1}-x_{n}\right\|=0 .
$$

We also obtain from the Definition of $w_{n}$ and (3.6) that

$$
\left\|w_{n}-x_{n}\right\|=\alpha_{n}\left\|x_{n}-x_{n-1}\right\| \leqslant\left\|x_{n}-x_{n-1}\right\| \rightarrow 0 \text { as } n \rightarrow \infty .
$$

By (3.6) and (3.7), we obtain

$$
\left\|w_{n}-x_{n+1}\right\|=\left\|w_{n}-x_{n}+x_{n}-x_{n+1}\right\| \leqslant\left\|w_{n}-x_{n}\right\|+\left\|x_{n}-x_{n+1}\right\| \rightarrow 0 \text { as } n \rightarrow \infty .
$$

Since $x_{n+1} \in C_{n+1}$, then

$$
\left\|u_{n}-x_{n+1}\right\|^{2} \leqslant\left\|w_{n}-x_{n+1}\right\|^{2}+\theta_{n} .
$$

From (3.8) and the fact that $\theta_{n} \rightarrow 0$ as $n \rightarrow \infty$, we obtain

$$
\lim _{n \rightarrow \infty}\left\|u_{n}-x_{n+1}\right\|=0 \text {. }
$$

Using (3.6) and (3.9), we have

$$
\left\|u_{n}-x_{n}\right\|=\left\|u_{n}-x_{n+1}+x_{n+1}-x_{n}\right\| \leqslant\left\|u_{n}-x_{n+1}\right\|+\left\|x_{n+1}-x_{n}\right\| \rightarrow 0 \text { as } n \rightarrow \infty .
$$

From (3.7) and (3.10), we get

$$
\left\|w_{n}-u_{n}\right\|=\left\|w_{n}-x_{n}+x_{n}-u_{n}\right\| \leqslant\left\|w_{n}-x_{n}\right\|+\left\|x_{n}-u_{n}\right\| \rightarrow 0 \text { as } n \rightarrow \infty .
$$


Now, Putting $u_{n}=T_{r_{n}} z_{n}, p \in \bigcap_{i=1}^{2} F\left(T_{i}\right) \cap E P(f, C)$ and using (2.1), we obtain

$$
\begin{aligned}
\left\|u_{n}-p\right\|^{2}= & \left\|T_{r_{n}} z_{n}-p\right\|^{2} \\
\leqslant & \left\|z_{n}-p\right\|^{2} \\
= & \left\|\left(1-\gamma_{n}\right)\left(T_{1}^{n} w_{n}-p\right)+\gamma_{n}\left(T_{2}^{n} y_{n}-p\right)\right\|^{2} \\
\leqslant & \left(1-\gamma_{n}\right)\left\|T_{1}^{n} w_{n}-p\right\|^{2}+\gamma_{n}\left\|T_{2}^{n} y_{n}-p\right\|^{2} \\
\leqslant & \left(1-\gamma_{n}\right)\left(1+k_{n, 1}\right)^{2}\left\|w_{n}-p\right\|^{2}+\gamma_{n}\left(1+k_{n, 2}\right)^{2}\left\|y_{n}-p\right\|^{2} \\
= & \left(1-\gamma_{n}\right)\left(1+k_{n, 1}\right)^{2}\left\|w_{n}-p\right\|^{2}+\gamma_{n}\left(1+k_{n, 2}\right)^{2}\left\|\left(1-\beta_{n}\right)\left(w_{n}-p\right)+\beta_{n}\left(T_{1}^{n} w_{n}-p\right)\right\|^{2} \\
= & \left(1-\gamma_{n}\right)\left(1+k_{n, 1}\right)^{2}\left\|w_{n}-p\right\|^{2}+\gamma_{n}\left(1+k_{n, 2}\right)^{2}\left[\left(1-\beta_{n}\right)\left\|w_{n}-p\right\|^{2}\right. \\
& \left.+\beta_{n}\left\|T_{1}^{n} w_{n}-p\right\|^{2}-\beta_{n}\left(1-\beta_{n}\right)\left\|w_{n}-T_{1}^{n} w_{n}\right\|^{2}\right] \\
\leqslant & \left(1-\gamma_{n}\right)\left(1+k_{n, 1}\right)^{2}\left\|w_{n}-p\right\|^{2}+\gamma_{n}\left(1+k_{n, 2}\right)^{2}\left[\left(1-\beta_{n}\right)\left\|w_{n}-p\right\|^{2}\right. \\
& \left.+\beta_{n}\left(1+k_{n, 1}\right)^{2}\left\|w_{n}-p\right\|^{2}-\beta_{n}\left(1-\beta_{n}\right)\left\|w_{n}-T_{1}^{n} w_{n}\right\|^{2}\right] \\
= & \left(1-\gamma_{n}\right)\left\|w_{n}-p\right\|^{2}+\left(1-\gamma_{n}\right)\left(2 k_{n, 1}+k_{n, 1}^{2}\right)\left\|w_{n}-p\right\|^{2} \\
& +\gamma_{n}\left(1+k_{n, 2}\right)^{2}\left[\left\|w_{n}-p\right\|^{2}+\beta_{n}\left(2 k_{n, 1}+k_{n, 1}^{2}\right)\left\|w_{n}-p\right\|^{2}-\beta_{n}\left(1-\beta_{n}\right)\left\|w_{n}-T_{1}^{n} w_{n}\right\|^{2}\right] \\
\leqslant & \left(1-\gamma_{n}\right)\left\|w_{n}-p\right\|^{2}+\gamma_{n}\left\|w_{n}-p\right\|^{2}+\left(1-\gamma_{n}\right)\left(2 k_{n, 1}+k_{n, 1}^{2}\right)\left\|w_{n}-p\right\|^{2} \\
& +\gamma_{n}\left(2 k_{n, 2}+k_{n, 2}^{2}\right)\left\|w_{n}-p\right\|^{2}+\gamma_{n}\left(1+k_{n, 2}\right)^{2} \beta_{n}\left(2 k_{n, 1}+k_{n, 1}^{2}\right)\left\|w_{n}-p\right\|^{2} \\
& -\gamma_{n} \beta_{n}\left(1-\beta_{n}\right)\left\|w_{n}-T_{1}^{n} w_{n}\right\|^{2} \\
= & \left\|w_{n}-p\right\|^{2}+\left(1-\gamma_{n}\right)\left(2 k_{n, 1}+k_{n, 1}^{2}\right)\left\|w_{n}-p\right\|^{2}+\gamma_{n}\left(2 k_{n, 2}+k_{n, 2}^{2}\right)\left\|w_{n}-p\right\|^{2} \\
& +\gamma_{n} \beta_{n}\left(1+k_{n, 2}\right)^{2}\left(2 k_{n, 1}+k_{n, 1}^{2}\right)\left\|w_{n}-p\right\|^{2}-\gamma_{n} \beta_{n}\left(1-\beta_{n}\right)\left\|w_{n}-T_{1}^{n} w_{n}\right\|^{2} \\
\leqslant & \left\|w_{n}-p\right\|^{2}+\left(1-\gamma_{n}\right)\left(2 k_{n, 1}+k_{n, 1}^{2}\right) M^{2}+\gamma_{n}\left(2 k_{n, 2}+k_{n, 2}^{2}\right) M^{2} \\
& +\gamma_{n} \beta_{n}\left(1+k_{n, 2}\right)^{2}\left(2 k_{n, 1}+k_{n, 1}^{2}\right) M^{2}-\gamma_{n} \beta_{n}\left(1-\beta_{n}\right)\left\|w_{n}-T_{1}^{n} w_{n}\right\|^{2} .
\end{aligned}
$$

Therefore,

$$
\begin{aligned}
\gamma_{n} \beta_{n}\left(1-\beta_{n}\right)\left\|w_{n}-T_{1}^{n} w_{n}\right\|^{2} \leqslant & \left\|w_{n}-p\right\|^{2}-\left\|u_{n}-p\right\|^{2}+\left(2 k_{n, 1}+k_{n, 1}^{2}\right) M^{2} \\
& +\gamma_{n}\left(2 k_{n, 2}+k_{n, 2}^{2}\right) M^{2}+\gamma_{n} \beta_{n}\left(1+k_{n, 2}\right)^{2}\left(2 k_{n, 1}+k_{n, 1}^{2}\right) M^{2} .
\end{aligned}
$$

Observe that, using (3.11), we have

$$
\begin{aligned}
\left\|w_{n}-p\right\|^{2}-\left\|u_{n}-p\right\|^{2} & =\left\|w_{n}\right\|^{2}-2\left\langle w_{n}, p\right\rangle+\|p\|^{2}-\left(\left\|u_{n}\right\|^{2}-2\left\langle u_{n}, p\right\rangle+\|p\|^{2}\right) \\
& =\left\|w_{n}\right\|^{2}-\left\|u_{n}\right\|^{2}-2\left\langle w_{n}-u_{n}, p\right\rangle \\
& \leqslant\left|\left\|w_{n}\right\|^{2}-\left\|u_{n}\right\|^{2}\right|+2\left|\left\langle w_{n}-u_{n}, p\right\rangle\right| \\
& \leqslant\left(\left\|w_{n}\right\|-\left\|u_{n}\right\|\right)\left(\left\|w_{n}\right\|+\left\|u_{n}\right\|\right)+2\left\|w_{n}-u_{n}\right\|\|p\| \\
& \leqslant\left\|w_{n}-u_{n}\right\|\left(\left\|w_{n}\right\|+\left\|u_{n}\right\|\right)+2\left\|w_{n}-u_{n}\right\|\|p\| \rightarrow 0 \text { as } n \rightarrow \infty
\end{aligned}
$$

Since $k_{n, i} \rightarrow 0$ as $n \rightarrow \infty$, for $i=1,2$, it follows from (3.12) and (3.13) that

$$
\lim _{n \rightarrow \infty} \gamma_{n} \beta_{n}\left(1-\beta_{n}\right)\left\|w_{n}-T_{1}^{n} w_{n}\right\|^{2}=0
$$

Using condition (C1), we get

$$
\lim _{n \rightarrow \infty}\left\|w_{n}-T_{1}^{n} w_{n}\right\|=0
$$

From the scheme (3.1) and (3.14), we get

$$
\left\|y_{n}-w_{n}\right\|=\beta_{n}\left\|T_{1}^{n} w_{n}-w_{n}\right\| \rightarrow 0 \text { as } \mathrm{n} \rightarrow \infty .
$$


Using (3.7) and (3.14), we obtain

$$
\left\|x_{n}-\mathrm{T}_{1}^{n} w_{n}\right\| \leqslant\left\|w_{n}-\mathrm{T}_{1}^{n} w_{n}\right\|+\left\|w_{n}-x_{n}\right\| \rightarrow 0 \text { as } \mathrm{n} \rightarrow \infty .
$$

Now,

$$
\begin{aligned}
\left\|x_{n}-T_{1}^{n} x_{n}\right\| & =\left\|x_{n}-T_{1}^{n} w_{n}+T_{1}^{n} w_{n}-T_{1}^{n} x_{n}\right\| \\
& \leqslant\left\|x_{n}-T_{1}^{n} w_{n}\right\|+\left\|T_{1}^{n} w_{n}-T_{1}^{n} x_{n}\right\| \\
& \leqslant\left\|x_{n}-T_{1}^{n} w_{n}\right\|+\left(1+k_{n, 1}\right)\left\|w_{n}-x_{n}\right\|,
\end{aligned}
$$

using (3.7) and (3.16), we get

$$
\lim _{n \rightarrow \infty}\left\|x_{n}-T_{1}^{n} x_{n}\right\|=0 .
$$

Therefore, from (3.6) and (3.17), we get

$$
\begin{aligned}
\left\|x_{n+1}-T_{1}^{n} x_{n+1}\right\| & \leqslant\left\|x_{n+1}-x_{n}\right\|+\left\|x_{n}-T_{1}^{n} x_{n}\right\|+\left\|T_{1}^{n} x_{n}-T_{1}^{n} x_{n+1}\right\| \\
& \leqslant\left(2+k_{n, 1}\right)\left\|x_{n+1}-x_{n}\right\|+\left\|x_{n}-T_{1}^{n} x_{n}\right\| \rightarrow 0 \text { as } n \rightarrow \infty .
\end{aligned}
$$

And

$$
\begin{aligned}
\left\|x_{n+1}-T_{1} x_{n+1}\right\| & =\left\|x_{n+1}+T_{1}^{n+1} x_{n+1}-T_{1}^{n+1} x_{n+1}-T_{1} x_{n+1}\right\| \\
& \leqslant\left\|x_{n+1}-T_{1}^{n+1} x_{n+1}\right\|+\left\|T_{1} x_{n+1}-T_{1}^{n+1} x_{n+1}\right\| \\
& =\left\|x_{n+1}-T_{1}^{n+1} x_{n+1}\right\|+\left\|T_{1} x_{n+1}-T_{1}\left(T_{1}^{n} x_{n+1}\right)\right\| \\
& \leqslant\left\|x_{n+1}-T_{1}^{n+1} x_{n+1}\right\|+\left(1+k_{1,1}\right)\left\|x_{n+1}-T_{1}^{n} x_{n+1}\right\| .
\end{aligned}
$$

Hence from (3.17) and (3.18), we get

$$
\lim _{n \rightarrow \infty}\left\|x_{n}-T_{1} x_{n}\right\|=0 .
$$

Similarly, from (2.1) and (3.3), we have

$$
\begin{aligned}
\left\|u_{n}-p\right\|^{2}= & \left\|T_{r_{n}} z_{n}-p\right\|^{2} \\
\leqslant & \left\|z_{n}-p\right\|^{2} \\
= & \left\|\left(1-\gamma_{n}\right)\left(T_{1}^{n} w_{n}-p\right)+\gamma_{n}\left(T_{2}^{n} y_{n}-p\right)\right\|^{2} \\
= & \left(1-\gamma_{n}\right)\left\|T_{1}^{n} w_{n}-p\right\|^{2}+\gamma_{n}\left\|T_{2}^{n} y_{n}-p\right\|^{2}-\gamma_{n}\left(1-\gamma_{n}\right)\left\|T_{1}^{n} w_{n}-T_{2}^{n} y_{n}\right\|^{2} \\
\leqslant & \left(1-\gamma_{n}\right)\left(1+k_{n, 1}\right)^{2}\left\|w_{n}-p\right\|^{2}+\gamma_{n}\left(1+k_{n, 2}\right)^{2}\left\|y_{n}-p\right\|^{2} \\
& -\gamma_{n}\left(1-\gamma_{n}\right)\left\|T_{1}^{n} w_{n}-T_{2}^{n} y_{n}\right\|^{2} \\
\leqslant & \left(1-\gamma_{n}\right)\left\|w_{n}-p\right\|^{2}+\left(2 k_{n, 1}+k_{n, 1}^{2}\right)\left\|w_{n}-p\right\|^{2}+\gamma_{n}\left(1+k_{n, 2}\right)^{2}\left[\left\|w_{n}-p\right\|^{2}\right. \\
& \left.+\beta_{n}\left(2 k_{n, 1}+k_{n, 1}^{2}\right)\left\|w_{n}-p\right\|^{2}\right]-\gamma_{n}\left(1-\gamma_{n}\right)\left\|T_{1}^{n} w_{n}-T_{2}^{n} y_{n}\right\|^{2} \\
= & \left(1-\gamma_{n}\right)\left\|w_{n}-p\right\|^{2}+\left(2 k_{n, 1}+k_{n, 1}^{2}\right)\left\|w_{n}-p\right\|^{2}+\gamma_{n}\left\|w_{n}-p\right\|^{2} \\
& +\gamma_{n}\left(2 k_{n, 2}+k_{n, 1}^{2}\right)\left\|w_{n}-p\right\|^{2}+\gamma_{n} \beta_{n}\left(1+k_{n, 2}\right)^{2}\left(2 k_{n, 1}+k_{n, 1}^{2}\right)\left\|w_{n}-p\right\|^{2} \\
& -\gamma_{n}\left(1-\gamma_{n}\right)\left\|T_{1}^{n} w_{n}-T_{2}^{n} y_{n}\right\|^{2} \\
= & \left\|w_{n}-p\right\|^{2}+\left(2 k_{n, 1}+k_{n, 1}^{2}\right)\left\|w_{n}-p\right\|^{2}+\gamma_{n}\left(2 k_{n, 2}+k_{n, 1}^{2}\right)\left\|w_{n}-p\right\|^{2} \\
& +\gamma_{n} \beta_{n}\left(1+k_{n, 2}\right)^{2}\left(2 k_{n, 1}+k_{n, 1}^{2}\right)\left\|w_{n}-p\right\|^{2}-\gamma_{n}\left(1-\gamma_{n}\right)\left\|T_{1}^{n} w_{n}-T_{2}^{n} y_{n}\right\|^{2} \\
\leqslant & \left\|w_{n}-p\right\|^{2}+\left(2 k_{n, 1}+k_{n, 1}^{2}\right) M^{2}+\gamma_{n}\left(2 k_{n, 2}+k_{n, 1}^{2}\right) M^{2} \\
& +\gamma_{n} \beta_{n}\left(1+k_{n, 2}\right)^{2}\left(2 k_{n, 1}+k_{n, 1}^{2}\right) M^{2}-\gamma_{n}\left(1-\gamma_{n}\right)\left\|T_{1}^{n} w_{n}-T_{2}^{n} y_{n}\right\|^{2} .
\end{aligned}
$$


Therefore,

$$
\begin{aligned}
\gamma_{n}\left(1-\gamma_{n}\right)\left\|T_{1}^{n} w_{n}-T_{2}^{n} y_{n}\right\|^{2} \leqslant & \left\|w_{n}-p\right\|^{2}-\left\|u_{n}-p\right\|^{2}+\left(2 k_{n, 1}+k_{n, 1}^{2}\right) M^{2} \\
& +\gamma_{n}\left(2 k_{n, 2}+k_{n, 1}^{2}\right) M^{2}+\gamma_{n} \beta_{n}\left(1+k_{n, 2}\right)^{2}\left(2 k_{n, 1}+k_{n, 1}^{2}\right) M^{2} .
\end{aligned}
$$

Therefore, using (3.13) and the fact that $k_{n, i} \rightarrow 0$ as $n \rightarrow \infty$, it follows from (3.20) that

$$
\lim _{n \rightarrow \infty} \gamma_{n}\left(1-\gamma_{n}\right)\left\|T_{1}^{n} w_{n}-T_{2}^{n} y_{n}\right\|^{2}=0
$$

From (C1), we have

$$
\lim _{n \rightarrow \infty}\left\|T_{1}^{n} w_{n}-T_{2}^{n} y_{n}\right\|^{2}=0
$$

Consequently,

$$
\lim _{n \rightarrow \infty}\left\|T_{1}^{n} w_{n}-T_{2}^{n} y_{n}\right\|=0
$$

Now,

$$
\begin{aligned}
\left\|x_{n}-T_{2}^{n} x_{n}\right\| & \leqslant\left\|x_{n}-T_{1}^{n} w_{n}\right\|+\left\|T_{1}^{n} w_{n}-T_{2}^{n} y_{n}\right\|+\left\|T_{2}^{n} y_{n}-T_{2}^{n} x_{n}\right\| \\
& \leqslant\left\|x_{n}-T_{1}^{n} w_{n}\right\|+\left\|T_{1}^{n} w_{n}-T_{2}^{n} y_{n}\right\|+\left(1+k_{n, 2}\right)\left\|y_{n}-x_{n}\right\| \\
& \leqslant\left\|x_{n}-T_{1}^{n} w_{n}\right\|+\left\|T_{1}^{n} w_{n}-T_{2}^{n} y_{n}\right\|+\left(1+k_{n, 2}\right)\left(\left\|y_{n}-w_{n}\right\|+\left\|w_{n}-x_{n}\right\|\right) .
\end{aligned}
$$

Using (3.7), (3.15), (3.16), and (3.21), we have

$$
\lim _{n \rightarrow \infty}\left\|x_{n}-T_{2}^{n} x_{n}\right\|=0
$$

Using (3.6) and (3.22), we obtain

$$
\begin{aligned}
\left\|x_{n+1}-T_{2}^{n} x_{n+1}\right\| & \leqslant\left\|x_{n+1}-x_{n}\right\|+\left\|x_{n}-T_{2}^{n} x_{n}\right\|+\left\|T_{2}^{n} x_{n}-T_{2}^{n} x_{n+1}\right\| \\
& \leqslant\left\|x_{n+1}-x_{n}\right\|+\left\|x_{n}-T_{2}^{n} x_{n}\right\|+\left(1+k_{n, 2}\right)\left\|x_{n}-x_{n+1}\right\| \\
& =\left(2+k_{n, 2}\right)\left\|x_{n}-x_{n+1}\right\|+\left\|x_{n}-T_{2}^{n} x_{n}\right\| \rightarrow 0 \text { as } n \rightarrow \infty .
\end{aligned}
$$

In similar fashion, we have

$$
\begin{aligned}
\left\|x_{n+1}-T_{2} x_{n+1}\right\| & =\left\|x_{n+1}+T_{2}^{n+1} x_{n+1}-T_{2}^{n+1} x_{n+1}-T_{2} x_{n+1}\right\| \\
& \leqslant\left\|x_{n+1}-T_{2}^{n+1} x_{n+1}\right\|+\left\|T_{2} x_{n+1}-T_{2}^{n+1} x_{n+1}\right\| \\
& =\left\|x_{n+1}-T_{2}^{n+1} x_{n+1}\right\|+\left\|T_{2} x_{n+1}-T_{2}\left(T_{2}^{n} x_{n+1}\right)\right\| \\
& \leqslant\left\|x_{n+1}-T_{2}^{n+1} x_{n+1}\right\|+\left(1+k_{1,2}\right)\left\|x_{n+1}-T_{2}^{n} x_{n+1}\right\| .
\end{aligned}
$$

Using (3.22) and (3.23), we obtain

$$
\lim _{n \rightarrow \infty}\left\|x_{n}-T_{2} x_{n}\right\|=0 .
$$

Step (iv): We show that $x^{*} \in \bigcap_{i=1}^{2} F\left(T_{i}\right) \cap E P(f, C)$.

Since $\left\{x_{n}\right\}$ is bounded, there exists a sub-sequence $\left\{x_{n_{j}}\right\}$ of $\left\{x_{n}\right\}$ such that $x_{n_{j}} \rightarrow x^{*} \in H$. Therefore from (3.19) and (3.24), it follows that $\lim _{j \rightarrow \infty}\left\|x_{n_{j}}-T_{i} x_{n_{j}}\right\|=0$, for $i=1$, 2. Consequently by Lemma 2.2, we have $T_{i} x^{*}=x^{*}$, for $i=1,2$. Thus, $x^{*} \in \bigcap_{i=1}^{2} F\left(T_{i}\right)$.

On the other hand,

$$
\left\|w_{n}-T_{2}^{n} y_{n}\right\| \leqslant\left\|T_{1}^{n} w_{n}-T_{2}^{n} y_{n}\right\|+\left\|w_{n}-T_{1}^{n} w_{n}\right\|
$$

Using (3.14) and (3.21), we get

$$
\lim _{n \rightarrow \infty}\left\|w_{n}-T_{2}^{n} y_{n}\right\|=0
$$


Using (3.14) and (3.25), we have

$$
\begin{aligned}
\left\|z_{n}-w_{n}\right\| & =\left\|\left(1-\gamma_{n}\right)\left(T_{1}^{n} w_{n}-w_{n}\right)+\gamma_{n}\left(T_{2}^{n} y_{n}-w_{n}\right)\right\| \\
& \leqslant\left(1-\gamma_{n}\right)\left\|T_{1}^{n} w_{n}-w_{n}\right\|+\gamma_{n}\left\|T_{2}^{n} y_{n}-w_{n}\right\| \rightarrow 0 \text { as } n \rightarrow \infty
\end{aligned}
$$

From (3.11) and (3.26), we obtain

$$
\left\|u_{n}-z_{n}\right\| \leqslant\left\|u_{n}-w_{n}\right\|+\left\|w_{n}-z_{n}\right\| \rightarrow 0 \text { as } n \rightarrow \infty .
$$

Therefore, since $r_{n} \geqslant a$, we get

$$
\lim _{n \rightarrow \infty} \frac{\left\|u_{n}-z_{n}\right\|}{r_{n}}=0
$$

Since $u_{n}=T_{r_{n}} z_{n}$, we obtain

$$
f\left(u_{n}, z\right)+\frac{1}{r_{n}}\left\langle z-u_{n}, u_{n}-z_{n}\right\rangle \geqslant 0, \quad \forall z \in C .
$$

From condition $\left(\mathrm{G}_{2}\right)$, we get

$$
\frac{1}{r_{n}}\left\langle z-u_{n}, u_{n}-z_{n}\right\rangle \geqslant f\left(z, u_{n}\right), \quad \forall z \in C,
$$

So that

$$
\left\langle z-u_{n_{j}}, \frac{u_{n_{j}}-z_{n_{j}}}{r_{n_{j}}}\right\rangle \geqslant f\left(z, u_{n_{j}}\right), \quad \forall z \in C .
$$

From $x_{n_{j}} \rightarrow x^{*}$, (3.10) and (3.27), we have $z_{n_{j}} \rightarrow x^{*}$ and $u_{n_{j}} \rightarrow x^{*}$. It follows from (3.28), (3.29), and conditon $\left(\mathrm{G}_{4}\right)$ that

$$
f\left(z, x^{*}\right) \leqslant 0, \quad \forall z \in C .
$$

Assume $s \in(0,1]$. For each $z \in \mathrm{C}$, let $z_{\mathrm{s}}=\mathrm{s} z+(1-\mathrm{s}) \mathrm{x}^{*}$. Then, $z_{\mathrm{s}} \in \mathrm{C}$ and so $\mathrm{f}\left(z_{\mathrm{s}}, x^{*}\right) \leqslant 0$. Hence, by $\left(\mathrm{G}_{1}\right)$ we have

$$
0=f\left(z_{s}, z_{s}\right) \leqslant \operatorname{sf}\left(z_{s}, z\right)+(1-s) f\left(z_{s}, x^{*}\right) \leqslant \operatorname{sf}\left(z_{s}, z\right)
$$

Dividing by s, we have

$$
f\left(z_{s}, z\right) \geqslant 0 \text {, i.e., } f\left(s z+(1-s) x^{*}, z\right) \geqslant 0, \forall z \in C .
$$

By taking limit as $s \rightarrow 0$ and $\left(\mathrm{G}_{3}\right)$, we get

$$
f\left(x^{*}, z\right) \geqslant 0, \quad \forall z \in C .
$$

Showing that $x^{*} \in \operatorname{EP}(f, C)$. Hence $x^{*} \in \bigcap_{i=1}^{2} F\left(T_{i}\right) \cap \operatorname{EP}(f, C)$. This shows that $\omega_{w}\left(x_{n}\right) \subset \bigcap_{i=1}^{2} F\left(T_{i}\right) \cap$ $\operatorname{EP}(f, C)$. Therefore, from (3.5) and Lemma 2.4, we obtain that $x_{n} \rightarrow x^{*}=P_{\bigcap_{i=1}^{2} F\left(T_{i}\right) \cap E P(f, C)} x_{0}$.

By Remark 1.1, Theorem 3.1 reduces to the following Corollary.

Corollary 3.2. Let $\mathrm{H}$ be a real Hilbert space and $\mathrm{C}$ be a nonempty closed, bounded and convex subset of $\mathrm{H}$. Let $f: C \times C \rightarrow \mathbb{R}$ be a bifunction satisfying conditions $\left(\mathrm{G}_{1}\right)-\left(\mathrm{G}_{4}\right)$. Let $\mathrm{T}_{i}: \mathrm{C} \rightarrow \mathrm{C}, \mathrm{i}=1,2$, be nonexpansive mapping such that $\bigcap_{i=1}^{2} \mathrm{~F}\left(\mathrm{~T}_{\mathrm{i}}\right) \cap \mathrm{EP}(\mathrm{f}, \mathrm{C}) \neq \emptyset$. Let $\left\{\mathrm{x}_{\mathrm{n}}\right\}$ be a sequence generated by $\mathrm{x}_{0}, \mathrm{x}_{1} \in \mathrm{C}$ and

$$
\left\{\begin{array}{l}
C_{0}=C, \\
w_{n}=x_{n}+\alpha_{n}\left(x_{n}-x_{n-1}\right), \\
y_{n}=\left(1-\beta_{n}\right) w_{n}+\beta_{n} T_{1} w_{n}, \\
z_{n}=\left(1-\gamma_{n}\right) T_{1} w_{n}+\gamma_{n} T_{2} y_{n}, \\
u_{n}=T_{r_{n}} z_{n}, \\
C_{n+1}=\left\{z \in C_{n}:\left\|u_{n}-z\right\|^{2} \leqslant\left\|w_{n}-z\right\|^{2}\right\} \\
x_{n+1}=P_{C_{n+1}} x_{0}, \quad \forall n \geqslant 0 .
\end{array}\right.
$$

Then, the sequence $\left\{x_{n}\right\}$ converges strongly to a point $x \in \bigcap_{i=1}^{2} F\left(T_{i}\right) \cap \operatorname{EP}(f, C)$, provided that $\left\{r_{n}\right\} \subset[a, \infty)$ for some $\mathrm{a}>0$ and the sequences of real numbers $\left\{\alpha_{n}\right\} \subset(0,1),\left\{\beta_{n}\right\}$, and $\left\{\gamma_{n}\right\}$ satisfy the following condition: 
(C1) $\beta_{n}, \gamma_{n} \in[\zeta, 1-\zeta]$, for some $\zeta \in(0,1)$.

Remark 3.3. Apart from extending the results of Phon-on et al. [27] from two nonexpansive mappings to two asymptotically nonexpansive mappings, our iterative sequence solves monotone equilibrium problem.

\section{Numerical example}

In this section, we give some example to illustrate Theorem 3.1 and justify that our scheme is implementable.

Consider $H=\mathbb{R}$ with its usual metric and let $C=[-10,10]$, which is clearly bounded closed and convex subset of $H$. Let $T_{i}: C \rightarrow C, i=1,2$, be defined by $T_{i}(x)=\frac{x}{2+i}$, then, $\forall x, y \in C, n \geqslant 1$, we have

$$
\left\|T_{i}^{n}(x)-T_{i}^{n}(y)\right\|=\left\|\frac{x}{(2+i)^{n}}-\frac{y}{(2+i)^{n}}\right\|=\frac{1}{(2+i)^{n}}\|x-y\| \leqslant\left(1+\frac{1}{(2+i)^{n}}\right)\|x-y\| .
$$

So, for each $i=1,2, T_{i}$ is asymptotically nonexpansive mapping with sequence $k_{n, i}=\frac{1}{(2+i)^{n}}, \quad \forall n \geqslant 1$ and $0 \in \cap_{i=1}^{2} F\left(T_{i}\right)$. Define a bifunction $f: C \times C \rightarrow \mathbb{R}$ by

$$
f(x, y)=4 y^{2}+x y-5 x^{2}, \forall x, y \in C .
$$

Then $f$ satisfies conditions $\left(G_{1}\right)-\left(G_{4}\right)$ and $0 \in E P(f, C)$. So, by Lemma 2.6, $T_{r_{n}} z$ is nonempty and singlevalued for each $x \in C$. Hence, there exists $x \in C$ such that

$$
f(x, y)+\frac{1}{r_{n}}\langle y-x, x-z\rangle \geqslant 0, \forall y \in C
$$

which is equivalent to

$$
4 y^{2}+x y-5 x^{2}+\frac{1}{r_{n}}\langle y-x, x-z\rangle \geqslant 0, \forall y \in C
$$

that is

$$
4 y^{2}+x y-5 x^{2}+\frac{1}{r_{n}}(y-x)(x-z) \geqslant 0, \forall y \in C,
$$

from which we obtain that

$$
4 r_{n} y^{2}+\left(r_{n} x+x-z\right) y+x z-\left(5 r_{n}+1\right) x^{2} \geqslant 0, \quad \forall y \in C
$$

Let $f(y)=4 r_{n} y^{2}+\left(r_{n} x+x-z\right) y+x z-\left(5 r_{n}+1\right) x^{2}$. Then, $f$ is a quadratic function of $y$ with coefficients $\mathrm{a}=4 \mathrm{r}_{\mathrm{n}}, \mathrm{b}=\mathrm{r}_{\mathrm{n}} x+x-z$, and $\mathrm{c}=x z-\left(5 \mathrm{r}_{\mathrm{n}}+1\right) x^{2}$. The discriminant $\Delta$ of $\mathrm{f}$ is given by

$$
\Delta=b^{2}-4 a c=\left(r_{n} x+x-z\right)^{2}-4\left(4 r_{n}\right)\left(x z-\left(5 r_{n}+1\right) x^{2}\right)=\left(x-z+9 r_{n} x\right)^{2} .
$$

We can see from the above that $f(y) \geqslant 0, \forall y \in C$. If it has at most one solution in $\mathbb{R}$, then $\Delta \leqslant 0$. Thus, we have $x=\frac{z}{1+9 r_{n}}$, i.e., $u_{n}=T_{r_{n}} z_{n}=\frac{z_{n}}{1+9 r_{n}}$.

Therefore if $\alpha_{n}=\frac{3}{10}$, then scheme (3.1) becomes

$$
\left\{\begin{array}{l}
w_{n}=x_{n}+\frac{3}{10}\left(x_{n}-x_{n-1}\right) \\
y_{n}=\left(1-\beta_{n}\right) w_{n}+\beta_{n} \frac{w_{n}}{3^{n}} \\
z_{n}=\left(1-\gamma_{n}\right) \frac{w_{n}}{3^{n}}+\gamma_{n} \frac{y_{n}}{4^{n}} \\
u_{n}=\frac{z_{n}}{1+9 r_{n}} \\
C_{n+1}=\left\{z \in C_{n}: z \leqslant \frac{u_{n}+w_{n}}{2}+\theta_{n}\right\} \\
x_{n+1}=P_{C_{n+1}} x_{0}, n \geqslant 0
\end{array}\right.
$$

where $\theta_{n}=\left(\left(2 k_{n, 1}+k_{n, 1}^{2}\right)\left(1+\gamma_{n} \beta_{n}\right)+\gamma_{n}\left(2 k_{n, 2}+k_{n, 2}^{2}\right)\left(1+\beta_{n}\left(2 k_{n, 1}+k_{n, 1}^{2}\right)\right)\right) M^{2}, M=\operatorname{diamC}=$ $\sup _{x, y \in C}\|x-y\|$. Let $\zeta=\frac{1}{10}, \beta_{n}=\gamma_{n}=\frac{1}{5}$, and $r_{n}=\frac{3}{40}$. From (4.1), using MATLAB, we obtain numerical results in Figures 1 and 2. 


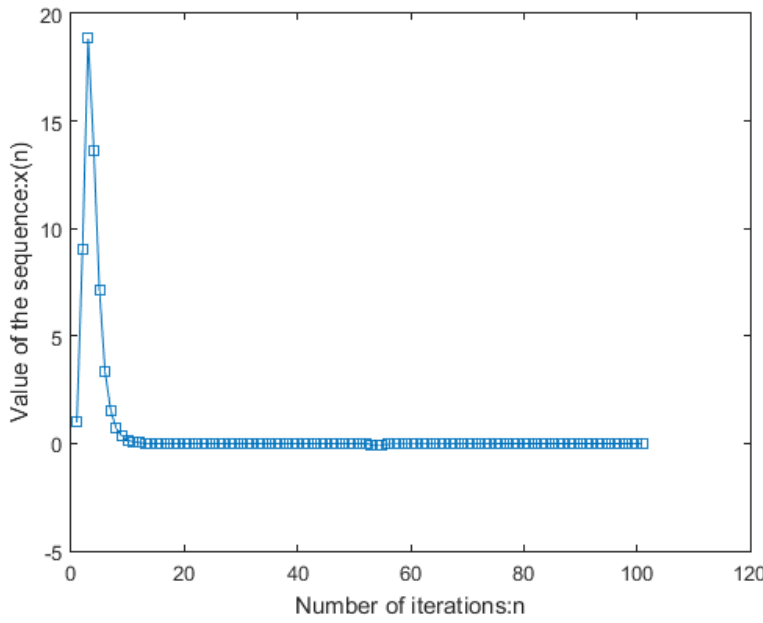

Figure 1: Convergence process of $\left\{x_{n}\right\}$ to 0 with initial points $x_{0}=1, x_{1}=9$.

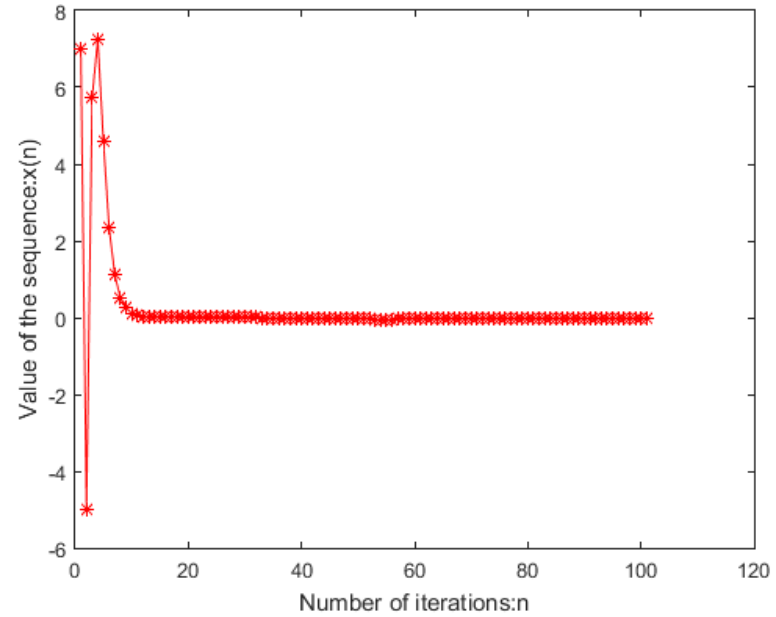

Figure 2: Convergence process of $\left\{x_{n}\right\}$ to 0 with initial points $x_{0}=7, x_{1}=-5$.

\section{Acknowledgment}

The authors thank the referees for their careful reading and vital observations which immensely improve presentation of the manuscript.

\section{References}

[1] R. P. Agarwal, D. O'Regan, D. R. Sahu, Iterative construction of fixed points of nearly asymptotically nonexpansive mappings, J. Nonlinear Convex Anal., 8 (2007), 61-79. 1

[2] P. K Ahn, D. V. Hieu, Parallel Hybrid Iterative Methods for Variational Inequalities, Equilibrium Problems, and Common Fixed Point Problems, Vietnam J. Math., 44 (2016), 351-374. 1

[3] B. Ali, M. H. Harbau, Convergence theorems for pseudomonotone equilibrium problem, split feasibility problem, and multivalued strictly pseudocontractive mappings, Numer. Funct. Anal. Optim., 40 (2019), 1194-1214.

[4] E. Blum, From optimization and variational inequalities to equilibrium problems, Math. Student, 63 (1994), 123-145. 1, $1,2.5$

[5] R. I. Bot, E. R. Csetnek, C. Hendrich, Inertial Douglas-Rachford splitting for monotone inclusion problems, Appl. Math. Comput., 256 (2015), 472-487. 1

[6] L. C. Ceng, A. Petrusel, X. Qin, J. C. Yao, Two inertial subgradient extragradient algorithms for variational inequalities with fixed-point constraints, Optimization, 70 (2021), 1337-1358.

[7] L. C. Ceng, A. Petrusel, C. F. Wen, J. C. Yao, Inertial-like subgradient extragradient methods for variational inequalities and fixed points of asymptotically nonexpansive and strictly pseudocontractive mappings, Mathematics, 7 (2019), 11 pages. 1

[8] P. L. Combettes, S. A. Hirstoaga, Equilibrium programming in hilbert spaces, J. Nonlinear Convex Anal., 6 (2005), 117-136. 2.6

[9] Q. L. Dong, H. B. Yuan, Y. J. Cho, T. M. Rassias, Modified inertial Mann algorithm and inertial CQ-algorithm for nonexpansive mappings, Optim. Lett., 12 (2018), 87-102. 1

[10] K. Goebel, W. A. Kirk, A fixed point theorem for asymptotically nonexpansive mappings, Proc. Amer. Math. Soc., 35 (1972), 171-174. 1, 1.1, 2.3

[11] K. Goebel, S. Reich, Uniform Convexity, Hyperbolic Geometry, and Nonexpansive Mappings, Marcel Dekker, New York, (1984). 2

[12] M. H. Harbau, Inertial hybrid self-adaptive subgradient extragradient method for fixed point of quasi- $\phi$-nonexpansive multivalued mappings and equilibrium problem, Adv. Theory Nonlinear Anal. Appl., 5 (2021), 507-522. 1

[13] M. H. Harbau, B. Ali, Hybrid linesearch algorithm for pseudomonotone equilibrium problem and fixed Points of Bregman quasi asymptotically nonexpansive mappings, J. Linear Topol. Algebra, 10 (2021), 153-177. 1

[14] D. V. Hieu, Y. J. Cho, Y.-B. Xiao, P. Kumam, Modified extragradient method for pseudomonotone variational inequalities in infinite dimensional Hilbert spaces, Vietnam J. Math., 49 (2020), 1165-1183. 1

[15] D. V. Hieu, L. D. Muu, P. K. Ahn, Parallel hybrid extragradient methods for pseudomonotone equilibrium problems and nonexpansive mappings, Numer. Algorithms, 73 (2016), 197-217. 1 
[16] D. V. Hieu, L. D. Muu, P. K. Quy, H. N. Duong, Regularization extragradient methods for equilibrium programming in Hilbert spaces, Optimization, 70 (2021), 1-31. 1, 1

[17] D. V. Hieu, J. J. Strodiot, L. D. Muu, Strongly convergent algorithms by using new adaptive regularization parameter for equilibrium problems, J. Comput. Appl. Math., 376 (2020), 21 pages. 1, 1

[18] S. A. Khan, K. R. Kazmi, D. Yambangwai, W. Cholamjiak, A hybrid projective method for solving system of equilibrium problems with demicontractive mappings applicable in image restoration problems, Math. Methods Appl. Sci., 43 (2020), 3413-3431. 1

[19] G. M. Korpelevich, The extragradient method for finding saddle points and other problems, Matecon, 12 (1976), 747-756. 1

[20] D. A. Lorenz, T. Pock, An inertial forward-backward algorithm for monotone inclusions, J. Math. Imaging Vision, 51 (2015), 311-325. 1

[21] P.-E. Maingé, Convergence theorems for inertial KM-type algorithms, J. Comput. Appl. Math., 219 (2008), 223-236. 1

[22] W. A. Mann, Mean value methods in iteration, Proc. Amer. Math. Soc., 4 (1953), 506-510. 1, 1

[23] C. Martinez-Yanes, H.-K. Xu, Strong convergence of the CQ method for fixed point processes, Nonlinear Anal., 64 (2006), 2400-2411. 2.4

[24] A. Moudafi, Second-order differential proximal methods for equilibrium problems, JIPAM. J. Inequal. Pure Appl. Math., 4 (2003), 7 pages. 1

[25] L. D. Muu, W. Oettli, Convergence of an adaptive penalty scheme for finding constrained equilibria, Nonlinear Anal., 18 (1992), 1159-1166. 1

[26] K. O. Oyewole, L. O. Jolaoso, C. Izuchuwu, O. T. Mewomo, On approximation of common solution of finite family of mixed equilibrium problems with $\mu-\eta$ relaxed monotone operator in a banach space, Politehn. Univ. Bucharest Sci. Bull. Ser. A Appl. Math. Phys., 81 (2019), 19-34. 1

[27] A. Phon-on, N. Makaje, A. Sama-Ae, K. Khongraphan, An inertial S-iteration process, Fixed Point Theory Appl., 2019 (2019), 14 pages. 1, 3.3

[28] E. Picard, Memoire sur la theorie des equations aux derivees partielles et la methode des approximations successives, J. Math. Pures Appl., 6 (1890), 145-210. 1, 1

[29] ' B.T. Polyak, Some methods of speeding up the convergence of iteration methods, U.S.S.R. Comput. Math. Math. Phys., 4 (1964), 1-17. 1

[30] D. R. Sahu, Applications of the S-iteration process to constrained minimization problems and split feasibility problems, Fixed Point Theory, 12 (2011), 187-204. 1

[31] M. J. Shang, Y. F. Su, X. L. Qin, A general iterative method for equilibrium problems and fixed point in Hilbert space, Fixed point Theory Appl., 2007 (2007), 9 pages. 1

[32] R. Suparatulatorn, W. Cholamjiak, S. Suantai, A modified S-iteration process for G-nonexpansive mappings in Banach spaces with graphs, Numer. Algorithms, 77 (2018), 479-490. 1, 1

[33] A. Tada, W. Takahashi, Strong convergence theorem for an equilibrium problem and a nonexpansive mapping, In: Nonlinear analysis and convex analysis, 2007 (2007), 609-617. 1

[34] A. Tada, W. Takahashi, Weak and strong convergence theorems for a nonexpansive mapping and equilibrium problem, J. Optim. Theory Appl., 133 (2007), 359-370.

[35] S. Takahashi, W. Takahashi, Viscosity approximation methods for equilibrium problems and fixed point problems in Hilbert spaces, J. Math. Anal. Appl., 331 (2007), 506-515. 1

[36] W. Takahashi, Y. Takeuchi, R. Kubota, Strong convergence theorems by hybrid methods for families of nonexpansive mappings in Hilbert spaces, J. Math. Anal. Appl., 341 (2008), 276-286. 1, 1

[37] W. Takahashi, K. Zembayashi, Strong and weak convergence theorems for equilibrium problems and relatively nonexpansive mappings in Banach spaces, Nonlinear Anal., 70 (2009), 45-57. 2.6

[38] K.-K. Tan, H. K. Xu, Fixed point iteration processes for asymptotically nonexpansive mappings, Proc. Amer. Math. Soc., 122 (1994), 733-739. 2.2 